\title{
Barrier Option Pricing Using Adjusted Transition Probabilities
}

\author{
G. Barone-Adesi, N. Fusari and J. Theal
}

\begin{abstract}
In the existing literature on barrier options much effort has been exerted to ensure convergence through placing the barrier in close proximity to, or directly onto, the nodes of the tree lattice. For a variety of barrier option types we show that such a procedure may not be a necessary prerequisite to achieving accurate option price approximations. Using the Kamrad and Ritchken (1991) trinomial tree model we show that with a suitable transition probability adjustment our "probability adjusted" model exhibits convergence to the barrier option price. We study the convergence properties of several option types including exponential barrier options, single linear time-varying barrier options, double linear timevarying barriers options and Bermuda options. For options whose strike price is close to the barrier we are able to obtain numerical results where other models and techniques typically fail. Furthermore, we show that it is possible to calculate accurate option price approximations with minimal effort for options with complicated barriers that defeat standard techniques. In no single case does our method require a repositioning of the pricing lattice nodes.
\end{abstract}

Keywords: barrier option, binomial tree, convergence rate, lattice models, option pricing, transition probability, trinomial tree

Introduction

Methods for pricing barrier options consist of two approaches: numerical based methods and theoretical expressions based on continuous-time models. In this paper we use a numerical technique with a suitable modification of the transition probabilities of the Kamrad and Ritchken (1991) trinomial tree ${ }^{1}$ to price several types of barrier options. In doing so, we illustrate the convergence of our method to the price, when available from an analytic solution, of the respective options. 
For European and American put and call options lattice models are able to yield convergence towards the option price given by a continuous-time model. In the case of a plain-vanilla option, convergence of the standard binomial or trinomial tree to the analytic value generally occurs within a few hundred time steps, yet due to distribution and non-linearity errors a persistent inaccuracy in the price of the option remains. If standard lattice methods are used to price more complex option types such as single constant barrier, multiple barrier and timevarying barrier options, they converge so slowly and exhibit such large bias that their use becomes impractical. This is an important observation because due to their lower cost and popularity in hedging financial and commodities positions, barrier and path-dependent options are now commonplace across all financial markets. Consequently, a need for improved lattice pricing models exists particularly for the more complex option types.

To explain the upward bias that occurs when pricing options using, for example, the CoxRoss-Rubinstein (CRR) binomial model, Boyle and Lau (1994) studied what happens to the price of a given option when the distance of the barrier to a layer of nodes in the binomial tree varies. They found that the upward bias problem arises as a consequence of the discretization of the nodes of the binomial lattice. Specifically, the bias occurs when the option barrier passes between two successive layers of nodes comprising the binomial tree without coming close to a node. Numerically this is perceived as a mispricing of the option that takes the form of an upward bias (i.e. convergence to the continuous-time price from above) added on to the option price. In order to reduce this bias, Boyle and Lau (1994) proposed to reposition the nodes in the binomial lattice such that the barrier passes as close as possible to a given layer of nodes in the binomial tree. With this modification, increased convergence rates were achieved; in one case Boyle and Lau report that convergence improved from 800 steps in the binomial tree to just 21 steps. This variation in the distance of the barrier from a layer of nodes manifests itself as an observable pattern in a plot of the option price against the number of time steps of the binomial lattice. One observes a series of alternating crests and troughs in a plot of the option price versus the number of time divisions in the tree. Accurate approximations to the continuous-time price occur at the troughs of the convergence graph where the barrier lies in close proximity to a given layer of the binomial lattice. Conversely, poor price approximations occur at the crests. 
It is known that the node-repositioning technique of Boyle and Lau is unable to produce an approximation to the option price when the initial underlying price lies close to the barrier, when there are multiple barriers or when the barrier is time-varying. Ritchken (1995) tries to address these particular problems by employing a trinomial lattice based upon the multinomial model of Kamrad and Ritchken (1991). Ritchken's method is also based upon a repositioning of the lattice nodes, however it differs from the method of Boyle and Lau in that Ritchken positions the lattice using a stretch-factor so that the barrier lies exactly upon a given level of the lattice nodes. Thus, despite increasing the number of time-divisions of the tree, there always exists a layer of nodes that coincides with the barrier level resulting in a rapid convergence to the continuous-time option price. While exhibiting good convergence, Ritchken's method suffers from three drawbacks. The first is that his method encounters difficulty converging to the continuous-time option price (and in some cases fails to converge at all) when the initial underlying price is very close to the barrier. The second drawback is that if a parameter of the option changes (maturity, volatility, etc...) then the entire lattice must be repositioned before calculating the new option price. Finally, Ritchken's method can only handle barrier options with constant boundaries.

Another efficacious pricing model for pricing barrier options is the adaptive mesh model (AMM) of Figlewski and Gao (1999). This technique works by grafting a fine resolution lattice onto the coarse lattice of the well-known trinomial tree and using smaller time and price increments to compute the finer mesh. In the case of a barrier option, the fine-resolution mesh can be grafted onto the coarse lattice nodes in the vicinity of the barrier to improve the price estimate. This has the effect of dramatically reducing the number of computations required to price the option in comparison to a normal trinomial tree model. It has the additional effect of implicitly determining the number of lattice levels required to achieve an accurate price approximation. Furthermore, layers of finer resolution mesh may be mutually overlaid to improve accuracy. However, owing to the difficulties in constructing a fineresolution lattice, the AMM model encounters problems with options possessing sloped linear and nonlinear barriers. Consequently, it would be of interest to develop a generalized latticepricing model that can be readily applied to options with arbitrarily specified barrier types.

Option pricing using lattice techniques can be thought of as a trade-off between convergence rates and having to reposition the nodes of the lattice in proximity to the barrier. In the following, we propose a simple modification applicable to both the CRR binomial 
model and the trinomial tree model. Specifically, we propose an adjustment to the transition probabilities that eliminates the need to reposition the nodes of the lattice. Simultaneously, this approach yields significantly increased convergence rates compared to alternative models. To calculate the continuous-time option price we make use of a series of analytical methods and empirical results available in the barrier option pricing literature. We are thus able to compare our calculations to a known value.

The remainder of the paper is organized as follows. Section I briefly reviews the Kamrad and Ritchken model of the trinomial tree. Section II explains the adjustment to the transition probabilities of the trinomial tree. In section III we present the results of our convergence rate analysis for single time-varying barrier options and compare the results of our calculations to other models taken from the barrier option literature. Section IV treats barrier options with multiple time-varying barriers and section V discusses exponential barriers. In section VI we apply the method to a Bermuda option and compare our model to others. Section VII provides some detail on computational complexity and time. In the final section we conclude.

\section{Pricing Using a Trinomial Lattice}

Appealing to the standard assumptions of lattice-based methods we assume that the price of the underlying asset follows a geometric Brownian motion process. Under this assumption the log-price drift is given by $\mu=r-\sigma^{2} / 2$ where $r$ is the risk-free rate and $\sigma$ is the instantaneous volatility at time $t$. The trinomial lattice for the underlying asset is first constructed using the up and down movement probabilities that are given by

$$
\begin{aligned}
& u=\exp (\sigma \sqrt{\delta t}) \\
& d=\frac{1}{u}=\exp (-\sigma \sqrt{\delta t})
\end{aligned}
$$

where $\sigma$ is the instantaneous volatility and $\delta t$ is the size of the time-division of the lattice tree. Starting from a known initial asset price, $S_{0}$, the underlying asset prices for the up, middle and down nodes at time $t+1$ are given by $u S_{0}, S_{0}$ and $d S_{0}$, respectively. A complete two-step trinomial lattice for the underlying asset is shown in Exhibit 1.

Exhibit 1 about here 
In the usual trinomial lattice implementation, after the creation of the underlying tree the option tree is constructed as in Exhibit 2.

\section{Exhibit 2 about here}

By working backwards through the option tree and appropriately discounting the calculated option prices one arrives at the lattice approximation to the option price. In such a trinomial tree, the option price evolves according to a set of lattice-specific probabilities such that (working backwards through the tree) the discounted option price, $C$, at time $t$ is given by

$$
C_{t}=e^{-r \delta t}\left(p_{u} C_{t+1, u}+p_{m} C_{t+1, m}+p_{d} C_{t+1, d}\right)
$$

where $p_{u}, p_{d}$ and $p_{m}$ are the risk-neutral transition probabilities of the trinomial lattice, $r$ is the risk-free rate and $C_{t+1, u}, C_{t+1, m}$ and $C_{t+1, d}$ are the call option prices for the up, middle and down nodes of the lattice, respectively. For the purposes of our implementation we use the following representation for the probabilities. Let

$$
\begin{gathered}
M=e^{r \delta t} \\
V=M^{2}\left(e^{\sigma^{2} \delta t}-1\right)
\end{gathered}
$$

then for the lattice probabilities we have:

$$
\begin{gathered}
p_{u}=\frac{\left(\left(V+M^{2}-M\right) e^{\lambda \sigma \sqrt{\delta t}}-(M-1)\right)}{\left(\left(e^{\lambda \sigma \sqrt{\delta t}}-1\right)\left(e^{2 \lambda \sigma \sqrt{\delta t}}-1\right)\right)} \\
p_{d}=\frac{\left(e^{2 \lambda \sigma \sqrt{\delta t}}\left(V+M^{2}-M\right)-e^{3 \lambda \sigma \sqrt{\delta t}}(M-1)\right)}{\left(\left(e^{\lambda \sigma \sqrt{\delta t}}-1\right)\left(e^{2 \lambda \sigma \sqrt{\delta t}}-1\right)\right)} \\
p_{m}=1-p_{u}-p_{d}
\end{gathered}
$$

where $\lambda$ is a trinomial lattice-specific parameter. ${ }^{2}$ It is clear that the greater the number of time divisions taken for the tree, the greater the accuracy of the final approximation to the true price. Traditionally, this method of pricing options has been used extensively for pricing European as well American plain-vanilla options. The adjusted transition probability method involves modifying these probabilities in order to produce accurate price approximation for the case of barrier options. Furthermore, using the adjusted probability method it is also relatively simple to extend the procedure to options with time-varying barriers as well as more exotic types like the Bermuda option. We now discuss the probability adjusted extension of the trinomial lattice method as applied to path-dependent barrier options. 
II. The Probability Adjusted Trinomial Tree Model for an Option with a Linear Time-Varying Barrier

A barrier option is a path-dependent option whose payoff is determined by whether the price of the underlying asset has reached some pre-determined price level negotiated at the time of the contract purchase. For example, in the case of a down-and-out ${ }^{3}$ barrier option, the option payoff is set to zero when the underlying price falls below the barrier. A barrier option like this can be priced using the same trinomial tree method used to price plain-vanilla options, however, the trinomial tree will converge at an extremely slow rate to the true price of the option. As discussed, it is possible to reposition the nodes of the lattice to increase convergence but this becomes difficult for curved barriers. Compared to repositioning techniques, the transition probability adjustment method provides a more intuitive way to increase the convergence rate. The basic methodology has been used previously to increase the convergence rates of Monte Carlo option pricing algorithms. It is known that, due to the discretized path along which the asset price evolves, it is possible for the underlying asset price to breach the option barrier without being detected by the Monte Carlo simulation as discussed in Geman and Yor (1996). One way to alleviate this problem is to use the supremum of a Brownian bridge to calculate the probability that the underlying asset price touches the barrier for any given step of the simulation. However, this method is not without its limitations. As noted in Baldi et al. (1999) this technique cannot be effectively used to price multiple barrier and time-varying barrier options. Consequently, Baldi derived a series of approximations for the exit probability of a Brownian bridge that can be used to price multiple and time-varying barrier options. Although he used these probabilities to improve upon Monte Carlo calculations, our contribution is to demonstrate that these probability approximations can be used to price options with single and multiple time-varying barriers on a simple trinomial lattice.

We describe our technique in the case of a down-and-out call option with a single linear time varying barrier. The same procedure applies to every type of barrier option, provided one knows the appropriate probability adjustment. 
Consider an option with a time-dependent, linear barrier. We define the barrier level $L_{t}$ (lower barrier at time $t$ ) using the equation of a line and choosing a slope, $m$, and an intercept, $b$, as in Equation (8)

$$
L_{t}=m t+b
$$

Note that the special case of a knock-out option with a constant barrier can be recovered by setting $m=0$ in Equation (8). The situation for the time-varying barrier is illustrated in Exhibit 3.

\section{Exhibit 3 about here}

The lattice of Exhibit 3 is indexed by the time division $\delta t=T / n$ where $T$ is the option maturity and $n$ is the number of divisions of the tree. Exhibit 4 shows a trinomial lattice with a time-varying linear barrier overlaid with two possible price paths labeled Diffusion Path 1 (DP1) and Diffusion Path 2 (DP2). These paths represent possible underlying price trajectories between times $t$ and $t+\delta t$ on the lattice. It can be seen that both DP1 and DP2 breach the barrier between adjacent node layers of the lattice and thus represent situations in which the option payoff should be set to zero.

\section{Exhibit 4 about here}

Paths such as these are analogous to the Monte Carlo paths that break the barrier yet remain undetected by the calculation. Because the trinomial lattice only approximates a continuoustime diffusion, diffusion paths 1 and 2 are possible in continuous time, but are not well approximated by the discretized nature of the lattice. Similarly, the trinomial lattice will possess paths like those indicated yet the stock price can end up above the barrier at the next time step of the tree. In such cases the crossing of the barrier is not detected. To account for this effect it is necessary to modify the option price calculation in order to reduce the expected payoff of the option by the total probability of all such possible diffusion paths. The probabilities of these diffusion paths are related to the exit probabilities calculated by Baldi et al. (1999).

We model a diffusion path of the logarithm of the stock price between lattice time divisions as a Brownian bridge. A Brownian bridge is a stochastic process in continuous-time whose 
probability distribution is given by the conditional distribution of a Weiner process. In particular, the starting and ending points of the process are known quantities.

Mathematically, a Brownian bridge is an $R^{\mathrm{n}}$-valued Gaussian process $\left(S_{t}\right)_{0 \leq t \leq T}$ for which we can define an exit probability, $\rho$. Let $\rho_{i}^{\delta t}$ be the probability that $S_{t}$ breaches the linear barrier, $i$ (with $i=L$ for the lower barrier and $i=U$ for the upper barrier), in the lattice time interval $[t, t+\delta t)$. More formally, it is the probability that the barrier is breached by a Brownian bridge that starts at $\log \left(S_{t}\right)$ at time $t$ and is conditional on it reaching $\log \left(S_{t+\delta t}\right)$ at time $t+\delta t$. In order to correct for the possibility that the diffusion path breaches the barrier we subtract this probability from unity so that we obtain the probability that the diffusion path does not breach the barrier in the interval $[t, t+\delta t)$. The correction is thereby obtained by multiplying the usual trinomial lattice probabilities by $\left(1-\rho_{i}^{\delta t}\right)$. This is what we imply by the phrase "transition probability adjustment".

We illustrate the method using a barrier option with a barrier defined as in equation (8). Using the subscript $L$ to denote a lower (i.e. down-and-out) barrier, the exit probability for this type of barrier is given by Baldi et al. (1999) as:

$$
\rho_{L}^{\delta t}\left(T_{0}, S_{T_{0}}, S_{T_{0}+\delta t}\right)=\exp \left[-\frac{2}{\sigma^{2}} \ln \left(\frac{S_{T_{0}}}{L_{T_{0}}}\right)\left(\frac{1}{\delta t} \ln \left(\frac{S_{T_{0}+\delta t}}{L_{T_{0}}}\right)+\frac{m}{b+m \delta t}\right)\right]
$$

where $S_{T_{0}}$ is the asset price at the current lattice node, $S_{T_{0}+\delta t}$ is the asset price at the next lattice node and $L_{T_{0}}$ is the barrier level at the current node. The numerical procedure involves correcting the lattice probabilities for the diffusion paths in the following manner. First we price the underlying tree using the normal trinomial tree method. At each node of the lattice we also calculate the level of the barrier associated with that particular node using the equation for the barrier, in this case equation (8). Subsequently, we work backwards through the option tree correcting for the exit probabilities, or rather we adjust the transition probabilities. At each node in the trinomial lattice there are three adjustments to perform for the "up", "mid" and "down" transitions. The calculation of the option price at a given node is shown in Equation (10).

$$
C\left(S_{T_{0}}\right)=e^{-r \delta t}\left(p_{u}\left(1-\rho_{L, u p}^{\delta t}\right) C\left(S_{T_{0+\delta \bar{t}}}^{u p}\right)+p_{m}\left(1-\rho_{L, \text { mid }}^{\delta t}\right) C\left(S_{T_{0+\delta \bar{t}}}^{\text {mid }}\right)+p_{d}\left(1-\rho_{L, d o w n}^{\delta t}\right) C\left(S_{T_{0+\delta \bar{t}}}^{\text {down }}\right)\right)(10)
$$

where $C\left(S_{T_{0}}\right)$ is the call option price corresponding to the node at which the barrier level is $L_{T_{0}}$ 
After iterating through the tree in this manner and performing the necessary adjustments we arrive at the final approximation to the option price.

\section{Options with a Single Linear Time-Varying Barrier}

To start with, we consider the special case of a single constant barrier option (by setting $m=0$ in Equation (8)). We study the behaviour of the probability adjustment method when the stock price approaches the barrier. Under these circumstances many lattice-based option valuation techniques have difficulty producing an approximation to the option price. In the case of the standard binomial tree, convergence is so slow that even after 5000 time divisions, there is significant difference between the approximate and analytical values. Even Ritchken's "stretched" trinomial tree method encounters difficulty in pricing a down-and-out call when the stock price approaches the barrier. For example, see Exhibit 5. Ritchken's method first encounters difficulty at 500 time-divisions and a stock price of 91.0. Furthermore, when the initial stock price is very close to the barrier, even as many as 5000 iterations are unable to provide an approximation to the option price.

\section{Exhibit 5 about here}

Conversely, because the probability adjustment technique is based on the lattice method with no node repositioning, we are always able to produce an approximation to the option price regardless of the stock price to barrier distance. Exhibit 5 presents our option price approximations in comparison to those of Ritchken's as the stock price approaches the barrier. At a stock value of 90.3, Ritchken's method is unable to produce an approximation to the price in 5000 divisions or less. Conversely, we obtain an approximation with 500 timedivisions. Furthermore, we are able to obtain price approximations when the stock price is extremely close to the barrier. Additionally, the quality of the approximation improves with decreasing distance between the stock price and barrier and, in the extreme case of a stock price of 90.01, we have very good agreement between our approximation and the analytical price for the adjusted trinomial model.

We now turn to the general case of a linear time varying barrier. To provide a numerical example we select some values for the slope and intercept of the barrier equation (8). We set 
$S_{0}=100, K=100, \sigma=25 \%, r=10 \%, T=1, m=10$ and $b=95$. In Exhibit 6 we plot the convergence curve for the linear barrier option with the parameters specified above.

\section{Exhibit 6 about here}

It is clear that the convergence of the adjusted tree is considerably faster than that of the normal (unadjusted) trinomial tree. Furthermore, the small fluctuations visible in the crests of the unadjusted curve are almost non-existent in the adjusted price curve. Unfortunately, for this case we do not possess an analytical solution to which we can compare our results.

\section{Options with Two Linear Time-Dependent Barriers}

Extending the example above, we now add an additional upper barrier, resulting in an option with two linear time-dependent barriers. Unlike the single barrier case, the probability adjustment must now account for both the upper and lower barriers. The appropriate exit probabilities for the upper, $U_{t}$, and lower, $L_{t}$, barrier were calculated by Baldi et al. (1999) and are given in Equation (11).

$$
\begin{aligned}
& \rho_{U}^{\delta t}\left(T_{0}, S_{T_{0}}, S_{T_{0+\delta \dot{t}}}\right)=\exp \left[-\frac{2}{\sigma^{2}} \ln \left(\frac{U_{T_{0}}}{S_{T_{0}}}\right)\left(\frac{1}{\delta t} \ln \left(\frac{U_{T_{0}}}{S_{T_{0+\delta t}}}\right)-\frac{m_{\text {upper }}}{b_{\text {upper }}+m_{\text {upper }} \delta t}\right)\right] \\
& \rho_{L}^{\delta t}\left(T_{0}, S_{T_{0}}, S_{T_{0+\delta i t}}\right)=\exp \left[-\frac{2}{\sigma^{2}} \ln \left(\frac{S_{T_{0}}}{L_{T_{0}}}\right)\left(\frac{1}{\delta t} \ln \left(\frac{S_{T_{0+\delta t}}}{L_{T_{0}}}\right)+\frac{m_{\text {lower }}}{b_{\text {lower }}+m_{\text {lower }} \delta t}\right)\right]
\end{aligned}
$$

where the previous variable definitions still apply and $U$ and $L$ are the values of the upper and lower barrier at time $\delta t$, respectively. To price the double barrier option the underlying tree is first constructed. For each time division of the lattice we calculate the level of both the upper and lower barriers using the equations that define the barrier. Once this is completed we can continue to price the option by calculating the option payoffs at maturity. These are calculated at the last time division of the lattice. We then work backwards through the tree calculating the option price at each given node using a slightly modified version of Equation (10) for the price of the option. If, at a given node, the underlying price is either above $U$ or below $\mathrm{L}$ we set the option value to zero, since the asset price has breached the barrier. Equation (12) gives the probability adjustment, $\rho$, for the double barrier option case. The 
adjustment is a linear combination of the exit probabilities defined in Equation (11) for the upper and lower transitions:

$$
\rho=1-\rho_{U}^{\delta t}-\rho_{L}^{\delta t}
$$

For the double time-varying barrier option it suffices to multiply the trinomial lattice probability by Equation (12) in order to effect the probability adjustment. To provide a numerical example, we choose the following set of parameters to describe the option: $S_{0}=100, K=100, \sigma=25 \%, r=10 \%, T=1, m_{\text {lower }}=-22, b_{\text {lower }}=92, m_{\text {upper }}=35$ and $b_{\text {upper }}=105$. Exhibit 7 shows the convergence curves for the double linear time-varying barrier option.

\section{Exhibit 7 about here}

We do not have an analytical value for the double time-varying barrier option either, yet it is clear that the plot converges to a constant value. The convergence is relatively stable after 1000 time divisions of the lattice and convergence is near-monotonic. The plot exhibits almost no periodical fluctuations in approaching a constant value for the option price. Notably, the unadjusted curve converges much more slowly to, presumably, the same value. However, an analytical or independent empirical result is needed to confirm the accuracy of our calculation.

To judge the goodness of our method we can consider the special case of a knock-out option with double constant barriers. Although there is no analytic formula available, this type of option has been widely investigated in the option pricing literature. Therefore, we can compare our results with other numerical approximations. The data presented in Exhibit 8 are taken from Pelsser (1997). It is clear from the table the probability-adjusted method is in good agreement with the values obtained using other methods in the existing barrier option pricing literature. Specifically we consider the Kunitomo and Ikeda (1992), finite difference and Pelsser (1997) methods. We conclude that for short-term maturity options, the model demonstrates accurate approximations to the option price within 2000 time-divisions of the lattice. Furthermore, these results do not differ significantly from those calculated using 1000 divisions of the tree.

Exhibit 8 about here 


\section{Options with Exponential Barriers}

We now turn our attention to the case of a barrier option with a time-varying exponential barrier. The probability adjustment is again given by Baldi et al. (1999) and is defined in Equation (13). This gives the exit probability in terms of the option parameters. As in the linear case, the barrier is characterized by its slope, $m$, intercept, $b$ and current lattice timestep, $T_{0}$ :

$$
L_{t}=\exp (m t+b)
$$

The probability correction is given by:

$$
\rho_{\exp }^{\delta t}\left(T_{0}, S_{T_{0}}, S_{T_{0+\delta t}}\right)=\exp \left[-\frac{2}{\sigma^{2}}\left(\ln \left(S_{T_{0}}\right)-b-m T_{0}\right)\left(\frac{\ln \left(S_{T_{0+\delta t}}\right)-b-m T_{0}}{\delta t}-m\right)\right]
$$

The parameters for the calculation are: $\mathrm{S}_{0}=95, \mathrm{~K}=100, \sigma=25 \%, \mathrm{r}=10 \%, \mathrm{~T}=1$ year, exponential barrier slope $=0.05$, exponential barrier intercept $=90$. The analytical value is 5.4861 .

Having specified the barrier and the parameters of the option we calculated the option price approximation for all time divisions from 1 to 1000 using the aforementioned procedure. The results are plotted in Exhibit 9.

\section{Exhibit 9 about here}

Because the exponential barrier option is priced using the same numerical technique that was applied to the single barrier option calculation times remain consistent and take approximately 4 seconds. After approximately 600 time-divisions of the tree, we note the decreased oscillations of the probability-adjusted model compared to that of the trinomial tree model. Convergence towards the analytical value of 5.4861 is also considerably faster. To judge the performance of our model for the exponential barrier option against another model, we compare our results to the empirical results published by Costabile (2002). The results are shown in Exhibit 10.

Exhibit 10 about here 
Usually, Costabile's method converges slightly faster than the adjusted probability technique due to the fact that he is repositioning the nodes of his lattice whereas our method does not. As mentioned, we know that methods based on lattice manipulation exhibit faster convergence. Nonetheless, Costabile's method is specific to single exponential barrier options only yet the adjusted method can handle a multitude of barrier types and is not restricted to a particular option type.

\section{Application to Bermuda Options}

A Bermuda option is a discretely monitored barrier option whose payoff is zero if the underlying price is below the barrier at some discretely monitored time, $t^{*}$. Exhibit 11 shows the diffusion paths that must be considered in order to apply the transition probability adjustment method.

\section{Exhibit 11 about here}

We can see two possible types of diffusion paths for the underlying price; a safe path and a knock-out path. Along the safe diffusion paths, the underlying price (even though discretely monitored) never falls below the barrier in continuous-time, yet in discrete time it appears to be a knock-out path. Looking now at the discretely monitored knock-out paths, the price of the underlying asset actually does fall below the barrier for some monitored time $t^{*}$. At this particular time instant the option does, in fact, knock-out which results in a payoff of zero. In applying the transition probability method the situations for both safe type paths and knockout type paths must be handled in order to achieve convergence to a fixed price. This is the basis of our method in the Bermuda option case.

The method for pricing a Bermuda option under transition probability adjustment proceeds as follows. First the underlying tree is constructed in the usual manner. We then determine the monitoring frequency of the option. This monitoring frequency provides us with a discrete set of times, $\left\{t^{*}\right\}$, at which we determine whether the barrier has or has not been breached. When these monitoring dates lie between the time partitions of the lattice we adjust the lattice probabilities. This situation is illustrated in Exhibit 12. 
In the downward transition from $S_{t}$ to $S_{t+1, d}$ the probability that the option expires is equal to the probability that a Brownian bridge between $S_{t}$ and $S_{t+1, d}$ falls, at time $t^{*}$, below the barrier. A complication arises when the barrier coincides with a time partition of the lattice as shown in Exhibit 13.

Exhibit 13 about here

In this case the exit probabilities cannot be calculated and the method suffers from discretization error. For the purpose of illustration, let the barrier level of Exhibit 11 be 95, the initial asset price, $S_{t}=100$ and $S_{t+1, d}=90$. In this scenario we would have to calculate the probability that the diffusion process falls below 95 at time $t^{*}$ having started at $S_{t}$ and ended at $S_{t+1, d}$. However, this probability equals unity when the barrier exceeds 90 . The underlying problem is that the option payoff on the trinomial tree is unaffected by the level of the barrier. Using the above example, the payoff of the option would be calculated as:

$$
C_{t}=e^{-r \bar{t}}\left(p_{u} C_{t+1, u}+p_{m} C_{t+1, m}+p_{d} 0\right)
$$

Equation (14) produces the same result for the price of the option, $C$, even if the barrier was placed at 90.01 since $C_{t+1, d}$ would still be zero. This result manifests itself as a large bias in the approximation to the option price.

To avoid this discretization problem we can restrict the computation of the trinomial tree, only to a number of steps such that the lattice time division does not coincide with barrier monitoring times. As a general guideline, in practice it is best to avoid choosing multiples of the prime factors of the monitoring frequency in order to eliminate this problem. Thus, if the monitoring frequency, $v$, is selected to be weekly and we assume 50 weeks in a year, it suffices to select a number of steps such as $\{51,53,57,59, \ldots\}$. Since the prime factors of 50 are 2 and 5, we do not compute the trinomial tree for steps that are multiples of 2 and 5.

The pricing of the Bermuda option is not significantly different from the single linear barrier case, the main difference being that a slight amount of additional effort is required to account for the discrete monitoring times as previously discussed. The probability adjustment is given by the cumulative normal distribution which is the probability distribution of a Brownian 
bridge between two successive points of the lattice. For the Weiner process $\{W\}_{t}$ that starts at $W\left(t_{1}\right)=\log \left(S_{t}\right)=a$ and ends at $W\left(t_{2}\right)=\log \left(S_{t+\delta t}\right)=b$, its distribution in time $t \in\left(t_{1}, t_{2}\right)$ is normal with mean:

$$
\mu=a+\frac{t-t_{1}}{t_{2}-t}(b-a)
$$

and variance

$$
\sigma^{2}=\frac{\left(t-t_{1}\right)\left(t_{2}-t\right)}{t_{2}-t_{1}} .
$$

The exit probability adjustment for the Bermuda option is therefore the cumulative normal distribution with mean, $\mu$, and variance, $\sigma^{2}$, as defined in Equations (15) and (16). The distribution function is evaluated at the log price of the underlying asset at the current lattice node. The option price is then calculated using the trinomial lattice and the procedure described for the previous option types. To illustrate, we provide several numerical examples and compare the results to existing techniques for Bermuda options in the literature.

Broadie and Glasserman (1997) derive a continuity correction for discretely monitored barrier options using the Reimann zeta function. This allows them to price a Bermuda option accurately, yet their method breaks down when the underlying price is close to the barrier or the barrier is time varying. A comparison between the adjusted transition probability method with the continuity correction method is given in Exhibit 14.

\section{Exhibit 14 about here}

The table shows the results of the adjusted probability method compared to the values published in Brodie et al. (1997) for the corrected continuous pricing method. The option parameters are $S_{0}=100, K=100, \sigma=30 \%, r=10 \%$, and $T=0.2$. The number of monitoring times for the corrected continuous method is 50 (with $T=0.2$ ) and for the adjusted method this translates into 250 annual monitoring points. These values correspond to daily monitoring of the option by assuming 250 trading dates per year. Values of the true price are taken from Brodie et al. and are calculated using a specialized trinomial tree as described in Broadie, Glasserman and Kou (1999). Both pricing methods perform well, but when the underlying price is close to the barrier, the probability-adjusted method has a much 
smaller value for the relative error illustrating its ability to accurately price options for which the initial underlying asset price is close to the option barrier.

Naturally, we can reduce the monitoring frequency and therefore reduce the number of probability adjustments in the trinomial lattice. In doing so we can compare the adjusted model to additional results by Broadie et al. (1997). Exhibit 15 shows the comparison of the model results for reduced monitoring frequency (the other parameters are equal to the previous example).

\section{Exhibit 15 about here}

The increased accuracy of the transition probability adjusted trinomial tree over the correctedcontinuous method is immediately evident when the underlying asset price is close to the barrier. The increase in the relative error of the corrected-continuous model in the $m=25$ case is pronounced even several price units away from the barrier.

To provide a graphical depiction of the convergence properties of the probability adjusted method applied to a Bermuda option, in Exhibit 16 we provide a series of convergence plots for various barrier levels.

\section{Exhibit 16 about here}

Panels (a) through (c) show convergence graphs for barrier levels, $L$, of 95, 99.5 and 99.9, respectively with weekly barrier monitoring. The remaining parameters are taken from Table 2 in Duan et al. (2003): $S_{0}=100, K=100, \sigma=20 \%, r=10 \%, T=0.5$ and $\lambda=1.2$. Panels (a)-(c) clearly show the convergence of the probability adjusted method to the analytic price which is shown on the plots as a straight line. Fluctuations about the true price are small and in most cases are within less than one-tenth of a unit of the option price. Some oscillations of increasing period do remain, however. Panel (d) shows the convergence plot for Example 1 as published in Ahn, Figlewski and Gao (1999). The parameters for this calculation are taken to be $S_{0}=40, K=40, \sigma=40 \%, r=4.88 \%, T=1, L=35$ and a single monitoring time at $T=1 / 2$. The convergence to the true price in this case is nearmonotonic and occurs rapidly. There are no noticeable oscillations in this particular case. 
Combined, panels (a)-(d) illustrate the flexibility of the method for pricing options of various maturities and various barrier levels; including barrier levels that are very close to the initial underlying asset price.

VII. Numerical Performance

All calculations were implemented in the $\mathrm{C}++$ programming language. Exhibit 17 shows the CPU time in seconds spent on the adjusted trinomial option tree and the CPU time in microseconds spent on the adjusted trinomial underlying tree. Also shown is the total combined CPU time of the calculation. All computations were performed on a $1.83 \mathrm{GHz}$ CPU with 2 GB of RAM.

Exhibit 17 about here

The CPU time spent on the underlying tree is negligible and on the order of microseconds. Consequently, it is evident that the bulk of the CPU time is spent on generating the option tree. In fact, total calculation time and CPU option tree time are indistinguishable on the figure. Calculation time increases rapidly after 4000 lattice levels and at 10000 lattice levels, the calculation time is approximately 47 seconds. A typical calculation for 1000 lattice divisions and a time-varying barrier requires approximately 4 seconds.

\section{Conclusion}

In this paper we have presented a generalized method for pricing numerous types of barrier options that is based on a simple modification of the trinomial tree model. With minimal effort, the calculations can readily be implemented on a binomial lattice with little to no change in accuracy. This also has the effect of decreasing calculation time owing to the reduced number of lattice paths. Additionally, the model can be applied to any option as long as the exit probability can be calculated (or approximated) in a closed-form. Our results for time-varying barrier options are promising and demonstrate good convergence properties towards the continuous-time price of the option. More specifically, we have shown that it is possible to produce option price approximations even when the initial underlying price is very close to the barrier, a result that is often difficult to obtain using alternative models in the 
literature. Furthermore, the model produces accurate price approximations for options with short maturity.

We have applied the transition probability method to several types of time-varying barrier options including those with exponential, single linear and double linear barriers. A lack of closed-form option valuation equations for some option types renders it difficult to precisely gauge the accuracy of our approximations in the case of the single linear and double linear time-varying barriers. Nonetheless, the price appears to converge to a fixed value based on the convergence plots. The accuracy of the option price approximations produced by the model dominates that of the Kamrad and Ritchken model while avoiding any repositioning of the lattice nodes. This makes it expedient and simple to implement even for complex option types.

Application of the model to discretely monitored Bermuda options also produces good results with no loss in pricing accuracy when the barrier level is close to the underlying asset price. Given its straightforward implementation, the model outperforms more complicated pricing models and convergence to the true price is near to monotonic, a desirable property for an asset pricing model.

While no single lattice based pricing model can be used to estimate option prices with arbitrary accuracy, a given model selected from the literature can be used either as a general purpose pricing model or as a specialized pricing model tailored to a specific option. What is important is that the practitioner understands the advantages and shortcomings of any particular option pricing model. 


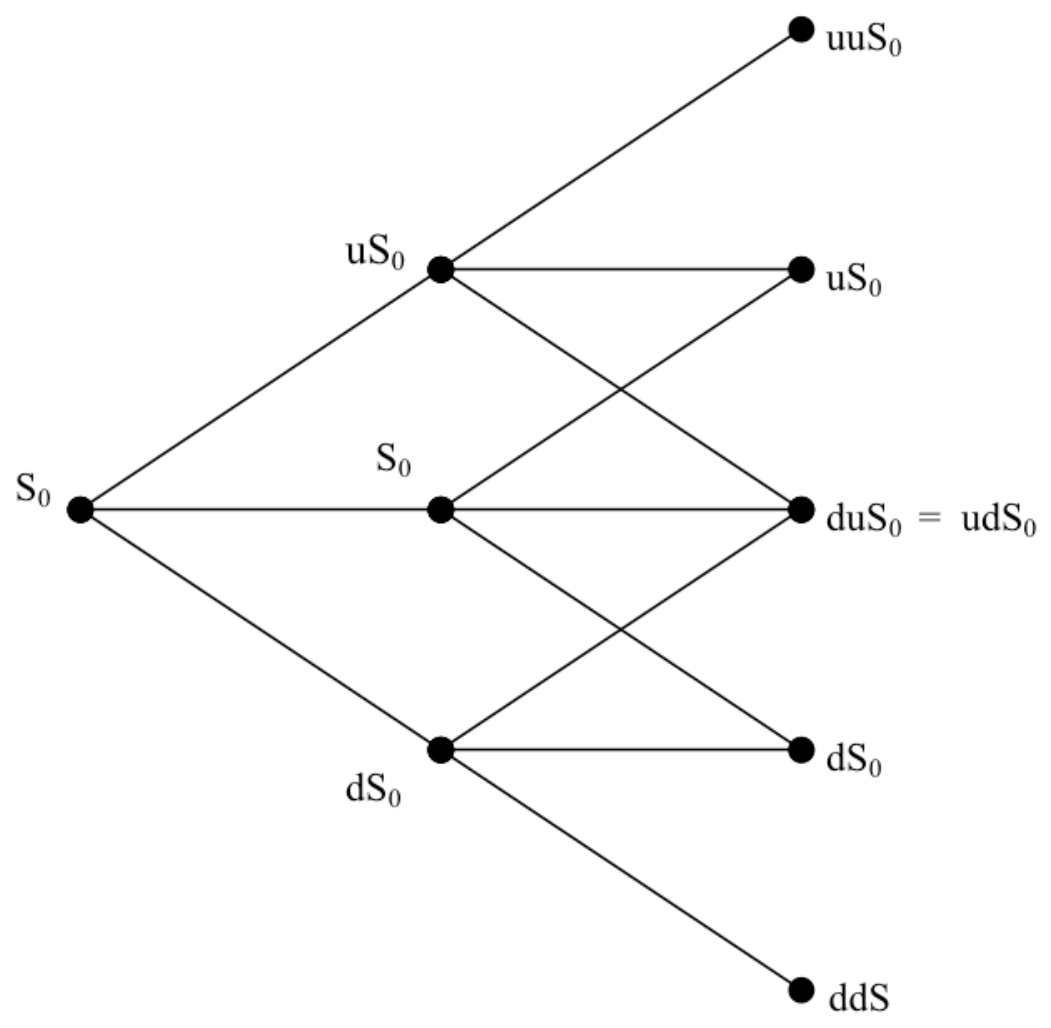

\section{Exhibit 1}

Evolution of the underlying trinomial tree. 


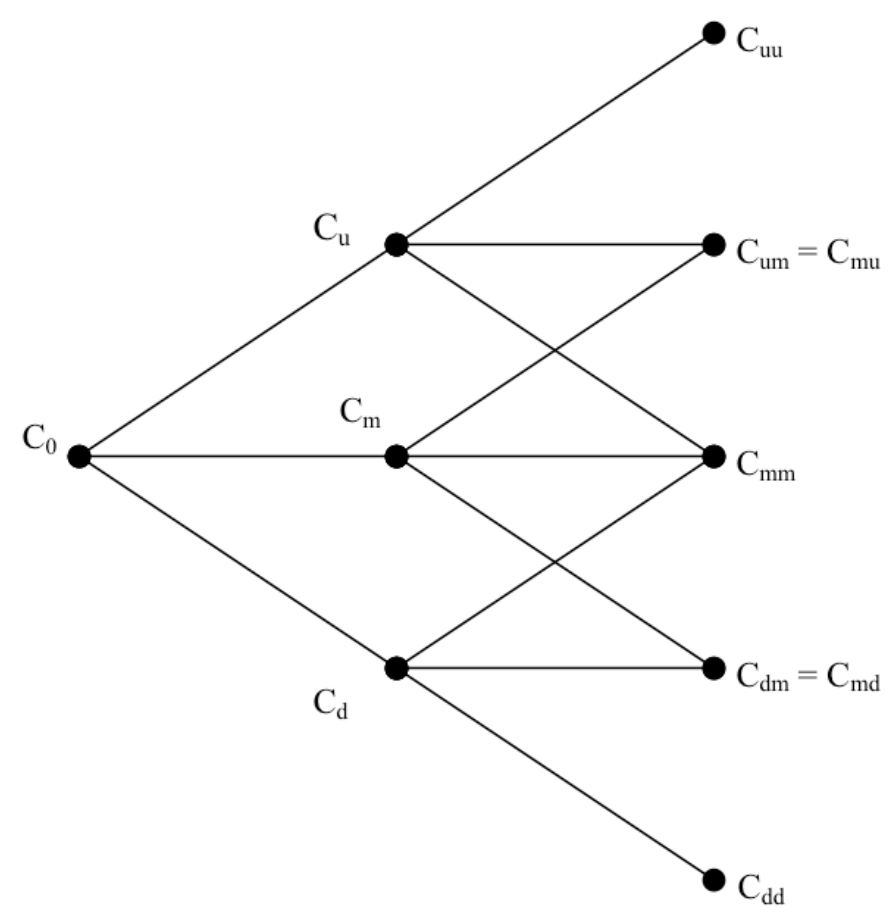

Exhibit 2

The option price trinomial lattice associated with the underlying asset tree. 


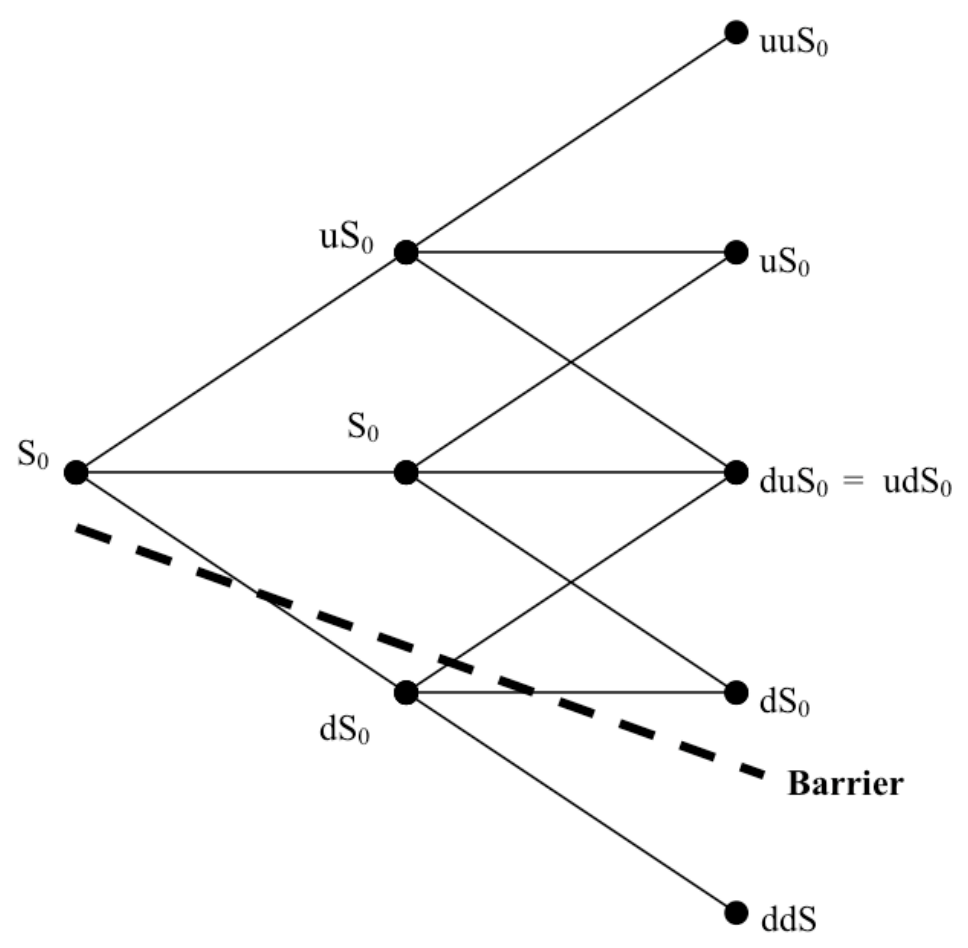

Exhibit 3

The trinomial lattice for an option with a linear time-varying barrier. 


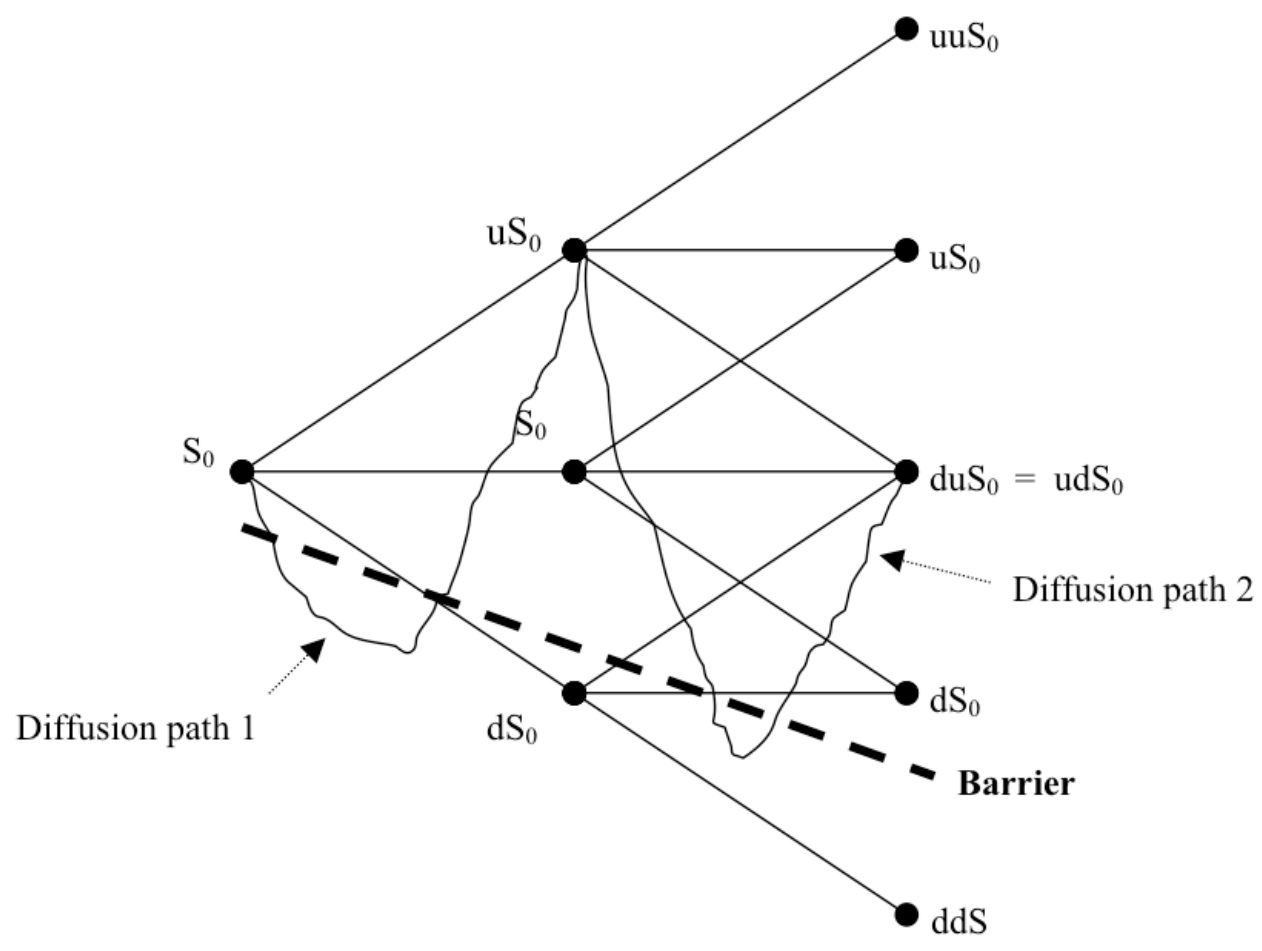

Exhibit 4

Trinomial lattice with diffusion paths that traverse the barrier between lattice nodes. 


\begin{tabular}{|c|c|c|c|c|c|c|c|}
\hline \multicolumn{8}{|c|}{ Number of Time Steps in the Tree } \\
\hline Stock Price & 500 & 1000 & 2000 & 3000 & 4000 & 5000 & $\begin{array}{c}\text { Analytic } \\
\text { Price }\end{array}$ \\
\hline 94.0 & $\begin{array}{c}4.849 / 4.723 \\
(4.863)\end{array}$ & $\begin{array}{c}4.814 / 4.839 \\
(4.864)\end{array}$ & $\begin{array}{c}4.866 / 4.811 \\
(4.864)\end{array}$ & $\begin{array}{c}4.840 / 4.880 \\
(4.864)\end{array}$ & $\begin{array}{c}4.839 / 4.845 \\
(4.864)\end{array}$ & $\begin{array}{c}4.82 / 4.833 \\
(4.864)\end{array}$ & 4.864 \\
\hline 93.0 & $\begin{array}{c}3.667 / 3.610 \\
(3.700)\end{array}$ & $\begin{array}{c}3.601 / 3.634 \\
(3.701)\end{array}$ & $\begin{array}{c}3.697 / 3.722 \\
(3.702)\end{array}$ & $\begin{array}{c}3.641 / 3.708 \\
(3.701)\end{array}$ & $\begin{array}{c}3.649 / 3.717 \\
(3.701)\end{array}$ & $\begin{array}{c}3.654 / 3.703 \\
(3.702)\end{array}$ & 3.702 \\
\hline 92.0 & $\begin{array}{c}2.45 / 2.526 \\
(2.504)\end{array}$ & $\begin{array}{c}2.509 / 2.398 \\
(2.506)\end{array}$ & $\begin{array}{c}2.487 / 2.425 \\
(2.506)\end{array}$ & $\begin{array}{c}2.507 / 2.497 \\
(2.506)\end{array}$ & $\begin{array}{c}2.488 / 2.502 \\
(2.506)\end{array}$ & $\begin{array}{c}2.454 / 2.464 \\
(2.506)\end{array}$ & 2.506 \\
\hline 91.5 & $\begin{array}{c}1.8 / 1.726 \\
(1.894)\end{array}$ & $\begin{array}{c}1.799 / 1.930 \\
(1.894)\end{array}$ & $\begin{array}{c}1.868 / 1.850 \\
(1.895)\end{array}$ & $\begin{array}{c}1.885 / 1.877 \\
(1.895)\end{array}$ & $\begin{array}{c}1.836 / 1.863 \\
(1.895)\end{array}$ & $\begin{array}{c}1.893 / 1.907 \\
(1.895)\end{array}$ & 1.895 \\
\hline 91.0 & $\begin{array}{c}1.222 / 1.333 \\
-\end{array}$ & $\begin{array}{c}1.176 / 1.155 \\
(1.274)\end{array}$ & $\begin{array}{c}1.239 / 1.286 \\
(1.274)\end{array}$ & $\begin{array}{c}1.221 / 1.248 \\
(1.275)\end{array}$ & $\begin{array}{c}1.275 / 1.217 \\
(1.275)\end{array}$ & $\begin{array}{c}1.224 / 1.272 \\
(1.274)\end{array}$ & 1.274 \\
\hline 90.5 & $\begin{array}{c}0.716 \text { / } 0.669 \\
-\end{array}$ & $\begin{array}{c}0.707 / 0.695 \\
-\end{array}$ & $\begin{array}{c}0.614 \text { / } 0.672 \\
-\end{array}$ & $\begin{array}{c}0.57 / 0.612 \\
-\end{array}$ & $\begin{array}{c}0.593 / 0.580 \\
(0.642)\end{array}$ & $\begin{array}{c}0.626 / 0.590 \\
(0.642)\end{array}$ & 0.642 \\
\hline 90.4 & $\begin{array}{c}0.561 / 0.521 \\
-\end{array}$ & $\begin{array}{c}0.576 / 0.544 \\
-\end{array}$ & $\begin{array}{c}0.549 / 0.558 \\
-\end{array}$ & $\begin{array}{c}0.498 / 0.542 \\
-\end{array}$ & $\begin{array}{c}0.462 \text { / } 0.512 \\
-\end{array}$ & $\begin{array}{c}0.458 / 0.480 \\
(0.515)\end{array}$ & 0.515 \\
\hline 90.3 & $\begin{array}{c}0.409 \text { / } 0.380 \\
-\end{array}$ & $\begin{array}{c}0.424 \text { / } 0.393 \\
-\end{array}$ & $\begin{array}{c}0.433 \text { / } 0.412 \\
-\end{array}$ & $\begin{array}{c}0.423 \text { / } 0.419 \\
-\end{array}$ & $\begin{array}{c}0.403 / 0.418 \\
-\end{array}$ & $\begin{array}{c}0.381 / 0.410 \\
-\end{array}$ & 0.387 \\
\hline 90.2 & $\begin{array}{c}0.263 \text { / } 0.248 \\
-\end{array}$ & $\begin{array}{c}0.271 / 0.252 \\
-\end{array}$ & $\begin{array}{c}0.281 / 0.260 \\
-\end{array}$ & $\begin{array}{c}0.287 \text { / } 0.267 \\
-\end{array}$ & $\begin{array}{c}0.289 \text { / } 0.273 \\
-\end{array}$ & $\begin{array}{c}0.288 \text { / } 0.276 \\
-\end{array}$ & 0.258 \\
\hline 90.1 & $\begin{array}{c}0.127 / 0.122 \\
-\end{array}$ & $\begin{array}{c}0.129 / 0.122 \\
-\end{array}$ & $\begin{array}{c}0.131 / 0.123 \\
-\end{array}$ & $\begin{array}{c}0.129 / 0.124 \\
-\end{array}$ & $\begin{array}{c}0.135 / 0.126 \\
-\end{array}$ & $\begin{array}{c}0.137 / 0.127 \\
-\end{array}$ & 0.129 \\
\hline 90.05 & $\begin{array}{c}0.063 \text { / } 0.061 \\
-\end{array}$ & $\begin{array}{c}0.063 \text { / } 0.061 \\
-\end{array}$ & $\begin{array}{c}0.064 \text { / } 0.061 \\
-\end{array}$ & $\begin{array}{c}0.064 \text { / } 0.061 \\
-\end{array}$ & $\begin{array}{c}0.064 \text { / } 0.061 \\
-\end{array}$ & $\begin{array}{c}0.065 \text { / } 0.061 \\
-\end{array}$ & 0.065 \\
\hline 90.01 & $\begin{array}{c}0.012 \text { / } 0.012 \\
\text { - }\end{array}$ & $\begin{array}{c}0.012 \text { / } 0.012 \\
\text { - }\end{array}$ & $\begin{array}{c}0.012 \text { / } 0.012 \\
\text { - }\end{array}$ & $\begin{array}{c}0.012 \text { / } 0.012 \\
-\end{array}$ & $\begin{array}{c}0.012 \text { / } 0.012 \\
-\end{array}$ & $\begin{array}{c}0.012 \text { / } 0.012 \\
-\end{array}$ & 0.013 \\
\hline
\end{tabular}

Exhibit 5

This table presents the results for the behaviour of the down-and-out call option price when the stock price is close to the barrier. The last column contains the price of the down-and-out call option using an analytical solution. The prices are displayed as adjusted binomial price / adjusted trinomial price. Prices in brackets are those given in Ritchken (1995). Prices indicated by ".-" were unable to be computed using the node repositioning method of Ritchken - that is the number of partitions used were insufficient to produce an approximation to the analytical price. 


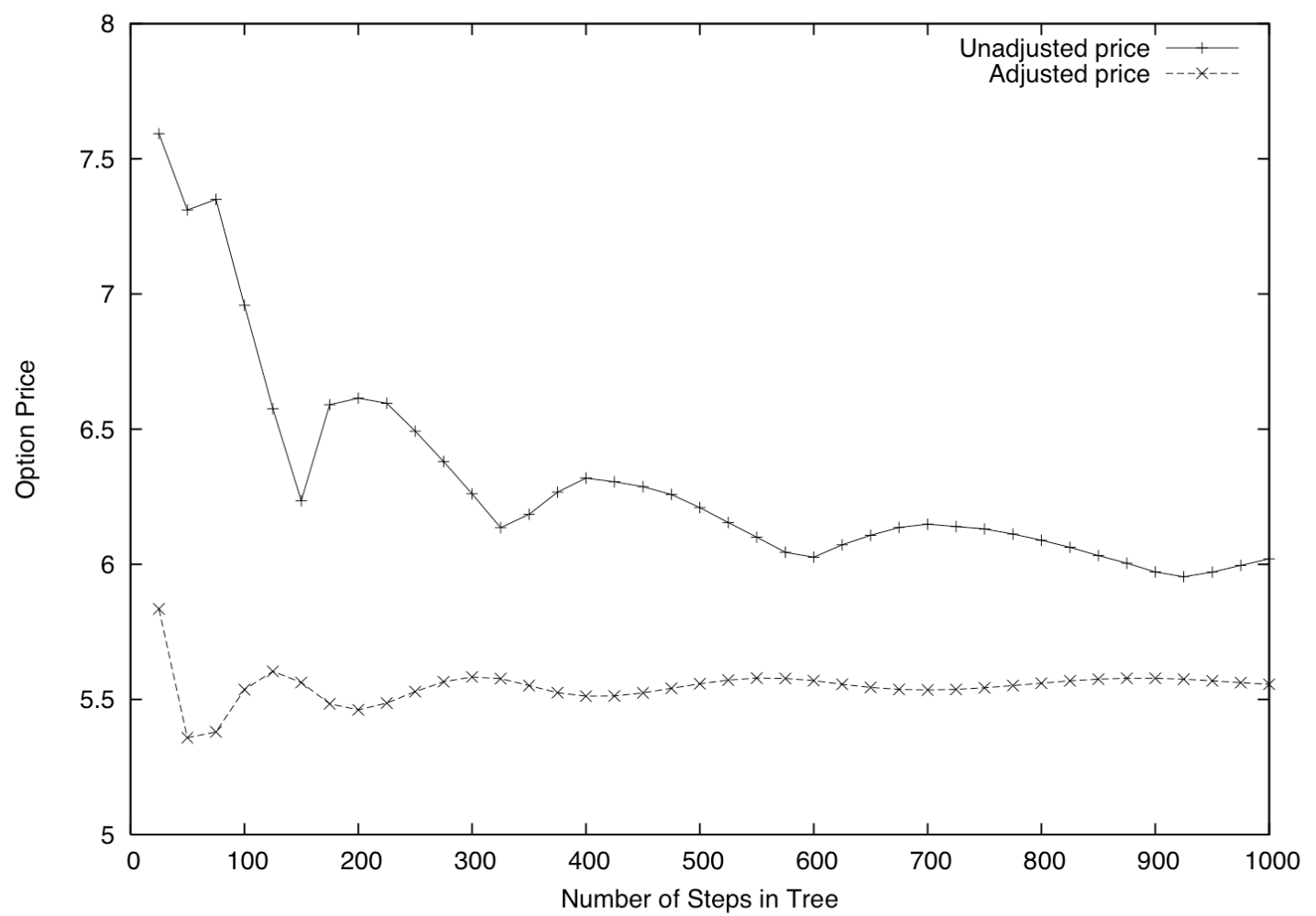

Exhibit 6

Convergence plot for the single linear time-varying barrier option. Option parameters are $\mathrm{S}_{0}=100, \mathrm{~K}=100, \sigma=25 \%, \mathrm{r}=10 \%, \mathrm{l}_{1}=10$ and $\mathrm{l}_{0}=95$. 


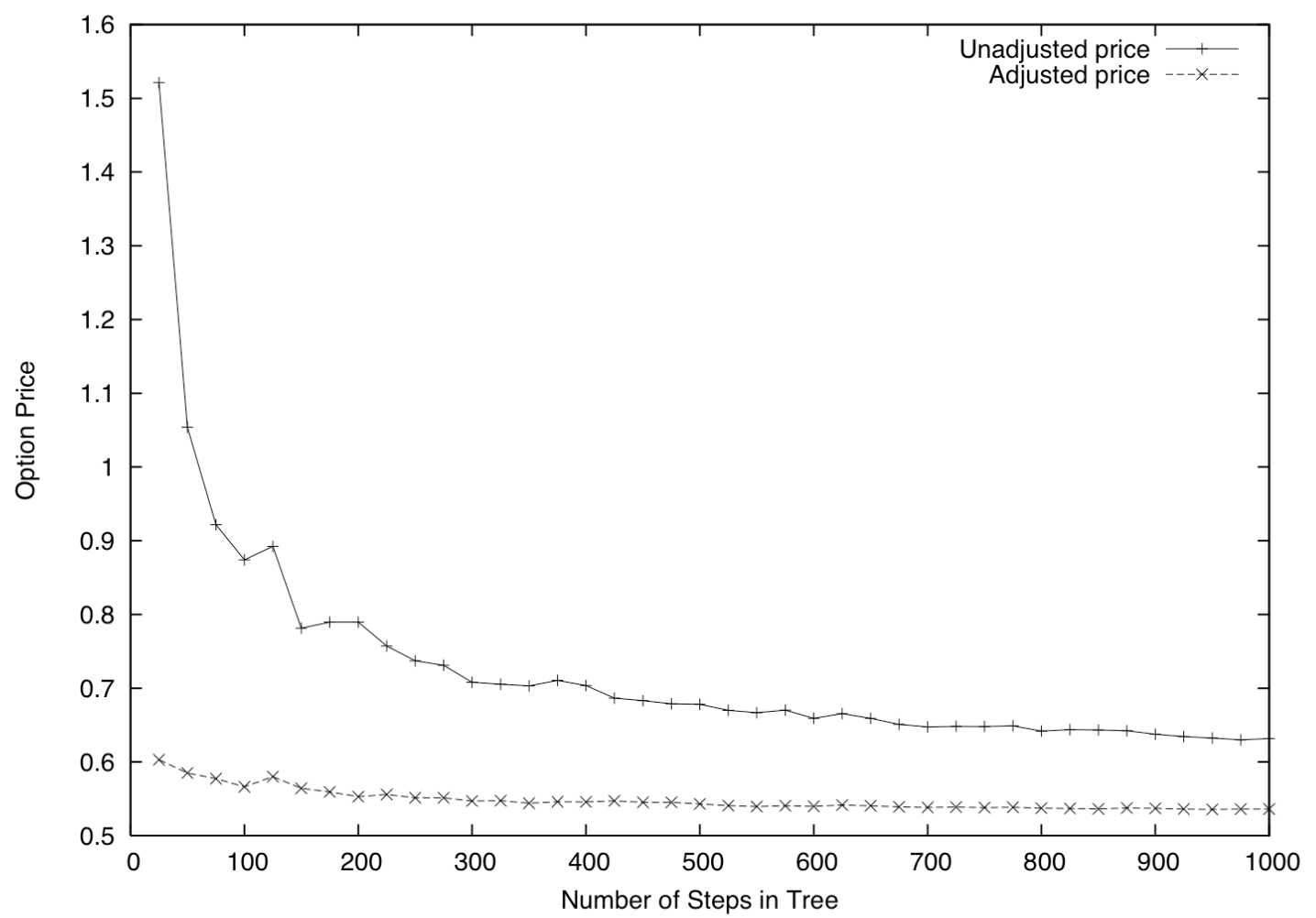

Exhibit 7

Convergence plot for the double linear barrier option. Option parameters are $\mathrm{S}_{0}=100, \mathrm{~K}=$ $100, \sigma=25 \%, \mathrm{r}=10 \%, \mathrm{l}_{1}=-22, \mathrm{l}_{0}=92, \mathrm{u}_{1}=35$ and $\mathrm{u}_{2}=105$. 


\begin{tabular}{|c|c|c|c|c|c|c|c|}
\hline \multicolumn{1}{|c}{ Vol } & \multicolumn{2}{c}{ U } & \multicolumn{2}{c}{ L } & \multicolumn{2}{c|}{ Ana } & \multicolumn{2}{c|}{ FD } & \multicolumn{2}{c|}{ Approx 1 } & Approx 2 \\
\cline { 2 - 8 }$\sigma=0.2$ & 1500 & 500 & 25.12 & 25.12 & 24.57 & $25.12 / 25.12$ & $25.12 / 25.12$ \\
& 1200 & 800 & 24.76 & 24.76 & 24.69 & $24.77 / 24.76$ & $24.76 / 24.76$ \\
& 1050 & 950 & 2.15 & 2.15 & 2.15 & $2.14 / 2.15$ & $2.15 / 2.15$ \\
\cline { 2 - 8 }$\sigma=0.3$ & 1500 & 500 & 36.58 & 36.58 & 36.04 & $36.59 / 36.58$ & $36.59 / 36.58$ \\
& 1200 & 800 & 29.45 & 29.45 & 29.40 & $29.42 / 29.40$ & $29.42 / 29.46$ \\
& 1050 & 950 & 0.27 & 0.27 & 0.27 & $0.27 / 0.26$ & $0.26 / 0.27$ \\
\cline { 2 - 8 }$\sigma=0.4$ & 1500 & 500 & 47.85 & 47.85 & 47.31 & $47.86 / 47.85$ & $47.85 / 47.84$ \\
& 1200 & 800 & 25.84 & 25.84 & 25.82 & $25.85 / 25.88$ & $25.83 / 25.86$ \\
& 1050 & 950 & 0.02 & 0.02 & 0.01 & $0.01 / 0.01$ & $0.01 / 0.01$ \\
\hline
\end{tabular}

\section{Exhibit 8}

Comparison of the adjusted method (binomial / trinomial) with values from Pelsser (1997). The option parameters used are $\mathrm{S}_{0}=1000, \mathrm{~K}=1000, \mathrm{r}=5 \%$ and $\mathrm{T}=1 / 12$. Note: "Approx 1" is calculated for 1000 time-divisions of the adjusted tree and "Approx 2" is calculated using 2000 time-divisions of the adjusted tree. Column "U" gives the upper barrier while column "L" gives the lower barrier. KI is calculated using the method of Kunitomo and Ikeda (1992), "Ana" are the results of Pelsser (1997) and "FD" is the finite difference calculation based on a 1000 by 1000 grid. 


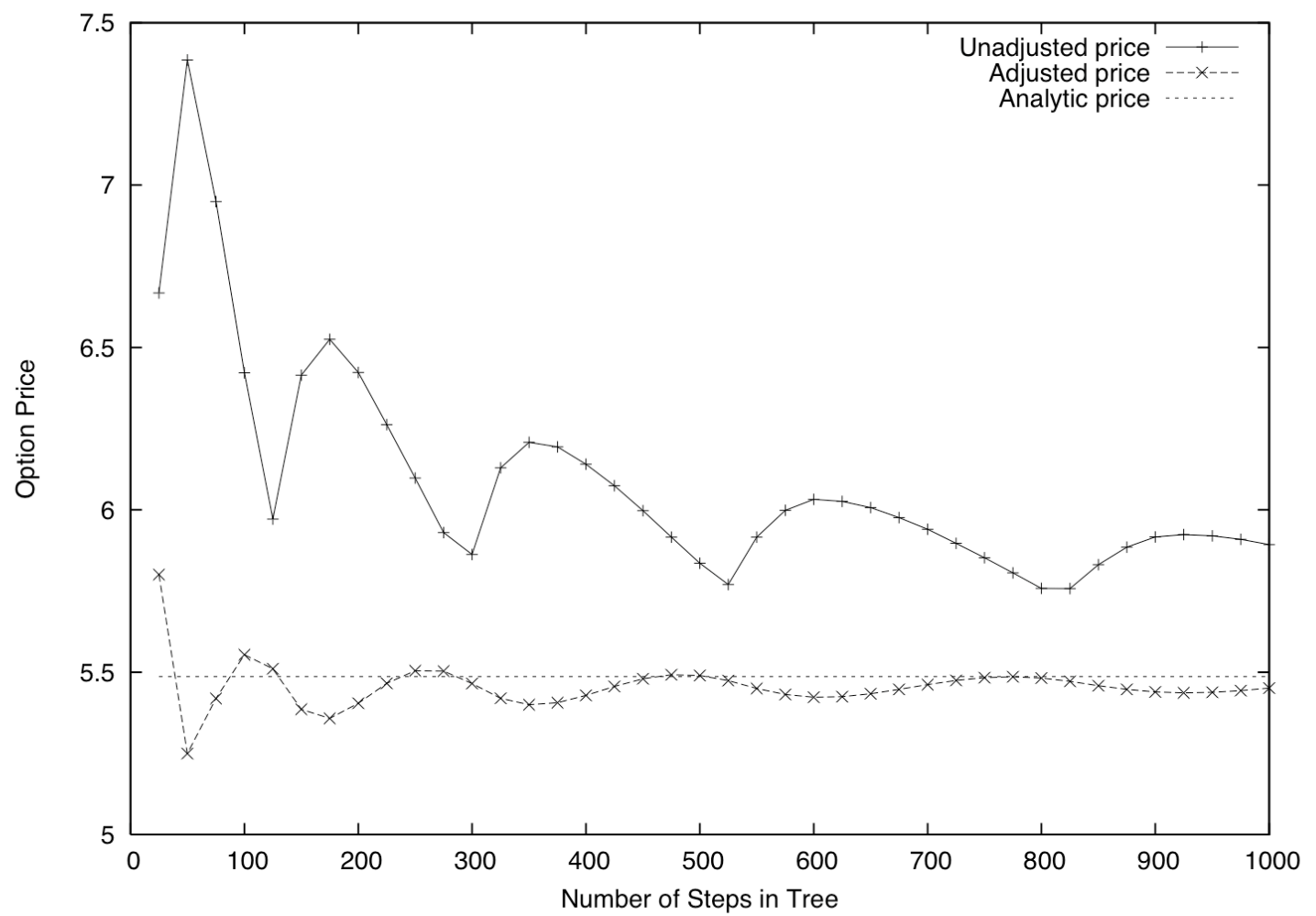

Exhibit 9

Results of the convergence calculation for an exponential barrier option. Parameters for the calculation are $\mathrm{S}_{0}=95, \mathrm{~K}=100, \sigma=25 \%, \mathrm{r}=10 \%, \mathrm{~T}=1$ year, exponential barrier slope $=$ 0.05 , exponential barrier intercept $=90$. The analytical value is 5.4861 . 


$$
\text { Slope }=m=-0.1
$$

Tree Lvls. Costabile Adjust

17

77

181

327

515

2100

4754

Analytic
7.002

6.631

6.753

6.787

6.786

6.802

6.868

6.889

6.896

$$
\text { Slope }=m=0.1
$$

$\begin{array}{ccc}\text { Tree Lvls. } & \text { Costabile } & \text { Adjusted } \\ 24 & 5.020 & 5.400 \\ 92 & 4.949 & 4.985 \\ 203 & 4.934 & 4.876 \\ 356 & 4.934 & 4.877 \\ 552 & 4.932 & 4.912 \\ 2174 & 4.929 & 4.915 \\ 4865 & 4.929 & 4.918 \\ \text { Analytic } & & 4.928\end{array}$

Exhibit 10

Comparison of results between the adjusted-probability method and the extended Cox-RossRubinstein method of Costabile (2002) 


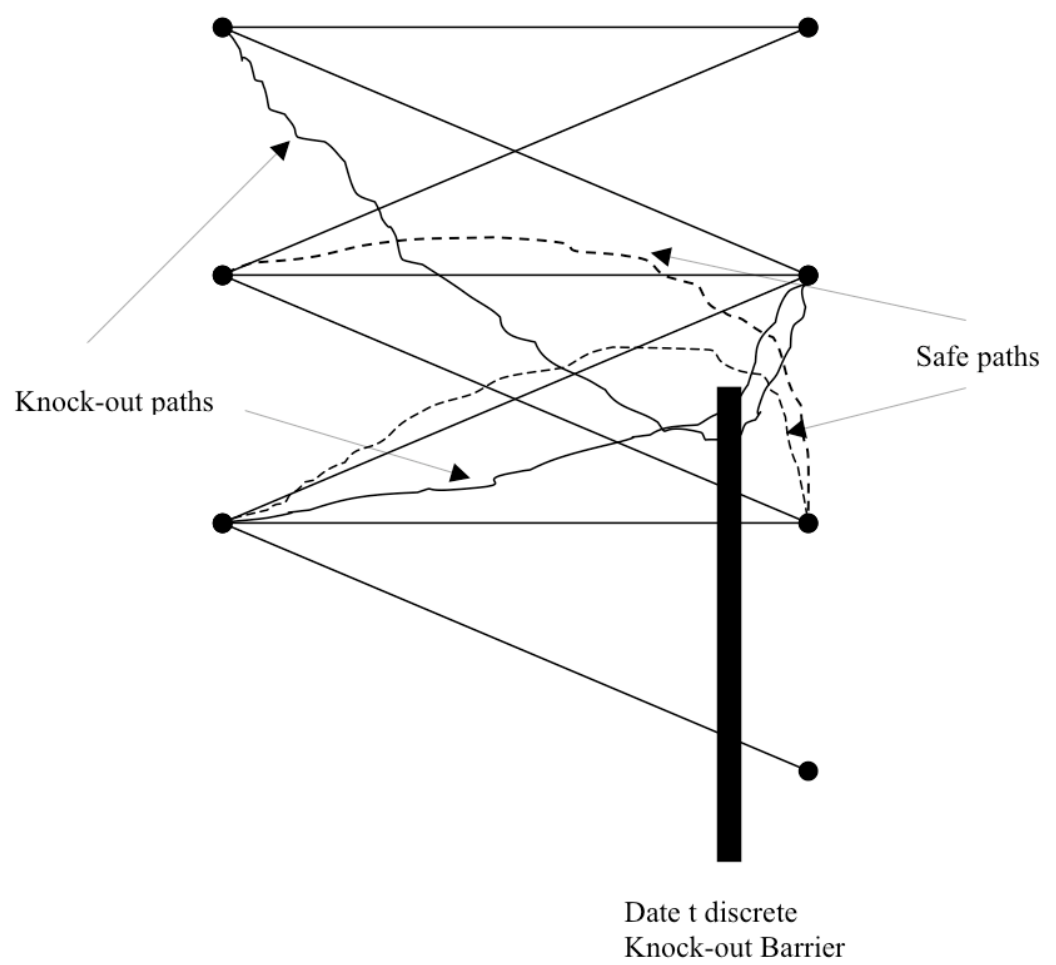

Exhibit 11

Diagram illustrating the two types of possible diffusion paths. These are denoted the knockout (continuous line) and safe type paths (dashed line). 


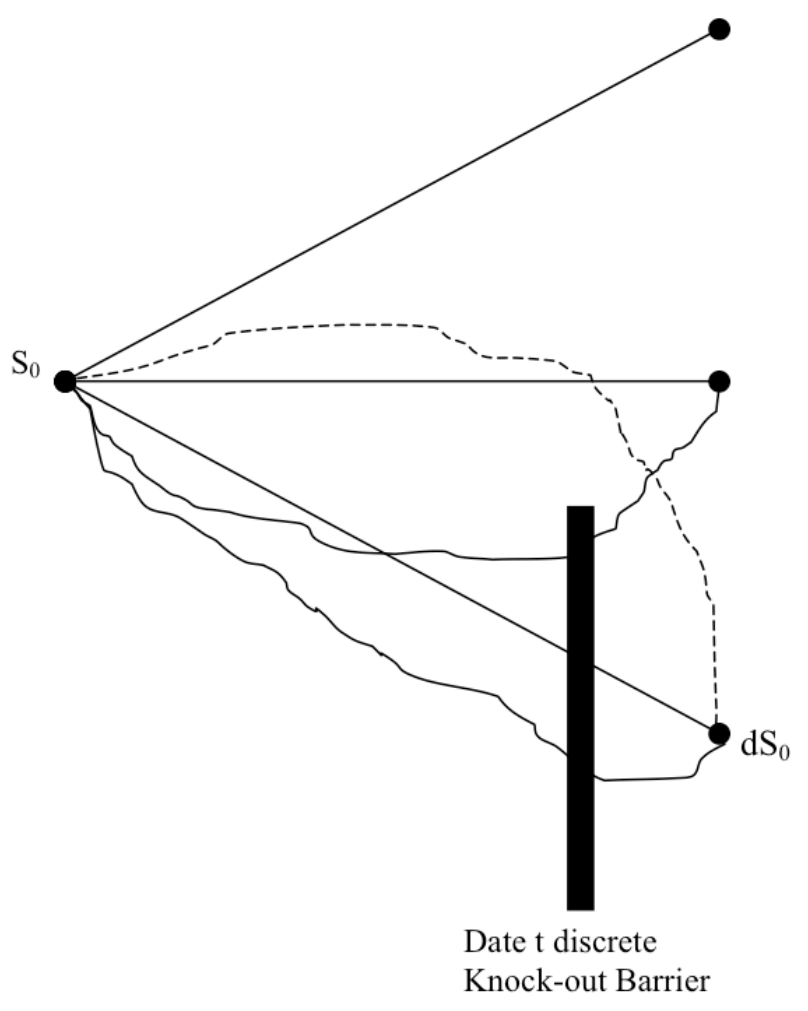

Exhibit 12

When the discrete monitoring times, $t^{*}$, lies between the lattice nodes, the probability correction is applied. 


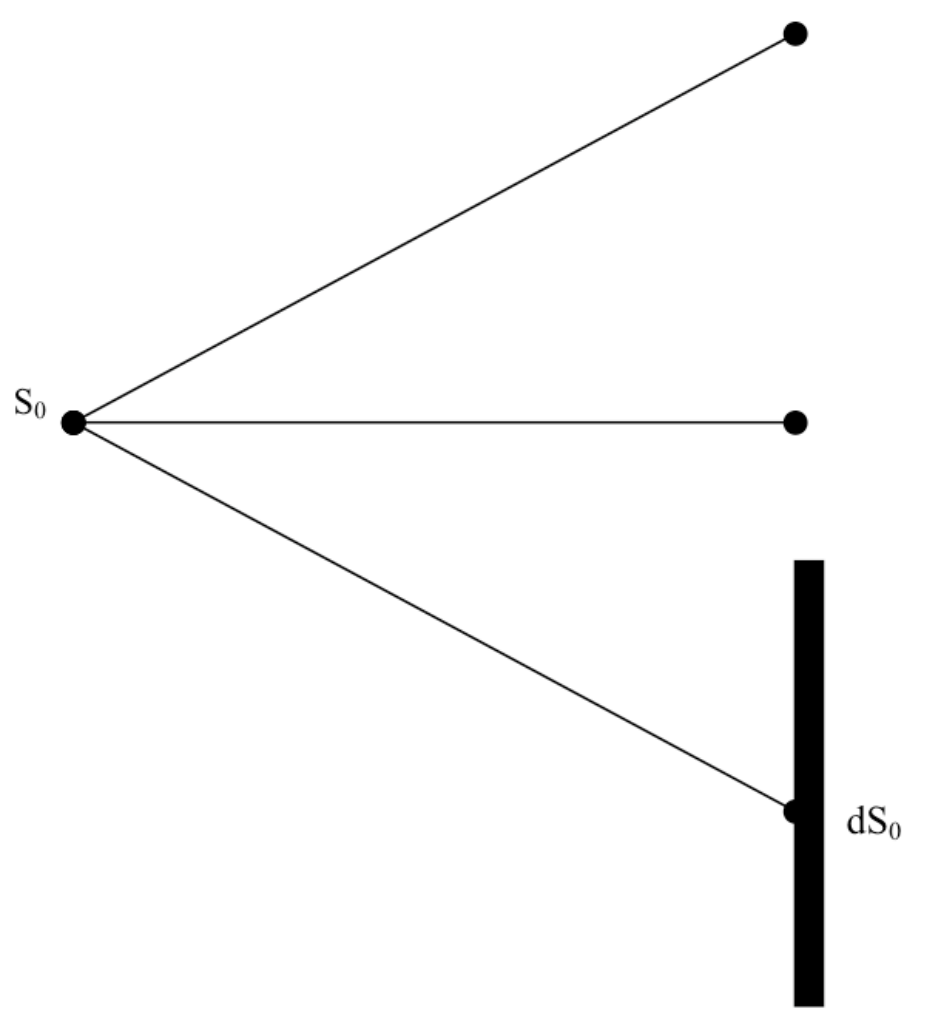

Date $t$ discrete

Knock-out Barrier

Exhibit 13

A complication arises when the barrier and a layer of lattice nodes coincide at a discrete monitoring time, $t^{*}$. 


\begin{tabular}{ccccccc}
\hline Barrier & $\begin{array}{c}\text { Continuous } \\
\text { Barrier }\end{array}$ & $\begin{array}{c}\text { Corrected } \\
\text { Continuous }\end{array}$ & $\begin{array}{c}\text { Probabililty } \\
\text { Adjusted }\end{array}$ & $\begin{array}{c}\text { True } \\
\text { Price }\end{array}$ & $\begin{array}{c}\text { Broadie } \\
\text { Rel. Err. (\%) }\end{array}$ & $\begin{array}{c}\text { Adj. } \\
\text { Rel. Err. (\%) }\end{array}$ \\
\hline 85 & 6.308 & 6.322 & 6.322 & 6.322 & 0 & 0 \\
86 & 6.283 & 6.306 & 6.306 & 6.306 & 0 & 0 \\
87 & 6.244 & 6.281 & 6.281 & 6.281 & 0 & 0 \\
88 & 6.185 & 6.242 & 6.242 & 6.242 & 0 & 0 \\
89 & 6.099 & 6.184 & 6.184 & 6.184 & 0 & 0 \\
90 & 5.977 & 6.098 & 6.097 & 6.098 & 0 & -0.02 \\
91 & 5.808 & 5.977 & 5.976 & 5.977 & 0 & -0.02 \\
92 & 5.579 & 5.810 & 5.811 & 5.810 & 0 & 0.02 \\
93 & 5.277 & 5.585 & 5.583 & 5.584 & 0.02 & -0.02 \\
94 & 4.888 & 5.288 & 5.283 & 5.288 & 0 & -0.09 \\
95 & 4.398 & 4.907 & 4.905 & 4.907 & 0 & -0.04 \\
96 & 3.792 & 4.428 & 4.430 & 4.427 & 0.02 & 0.07 \\
97 & 3.060 & 3.836 & 3.841 & 3.834 & 0.05 & 0.18 \\
98 & 2.189 & 3.121 & 3.131 & 3.126 & -0.16 & 0.16 \\
99 & 1.171 & 2.271 & 2.337 & 2.337 & -2.82 & 0 \\
\hline
\end{tabular}

\section{Exhibit 14}

Comparison between the results of Broadie and Glasserman (1997) and the adjusted transition probability method for a down-and-out call Bermudan style option. The option parameters are $S_{0}=100, K=100, \sigma=30 \%, r=10 \%$, and $T=0.2$. The number of monitoring times for the corrected continuous method is 50 (with $T=0.2$ ) and for the adjusted method this translates into 250 annual monitoring points. 


\begin{tabular}{ccccccc}
\hline & Barrier & $\begin{array}{c}\text { Corrected } \\
\text { Continuous }\end{array}$ & $\begin{array}{c}\text { Adjusted } \\
\text { Tree }\end{array}$ & True & $\begin{array}{c}\text { Cor. Cont. } \\
\text { Rel. Err. (\%) }\end{array}$ & $\begin{array}{c}\text { Adj. Tri. } \\
\text { Rel. Err. (\%) }\end{array}$ \\
\hline $\mathrm{m}=125$ & 85 & 6.327 & 6.326 & 6.326 & 0.02 & 0.01 \\
& 87 & 6.293 & 6.292 & 6.292 & 0.02 & 0.01 \\
& 89 & 6.210 & 6.210 & 6.210 & 0.00 & 0.00 \\
& 91 & 6.033 & 6.031 & 6.032 & 0.02 & -0.01 \\
& 93 & 5.688 & 5.686 & 5.688 & 0.00 & -0.03 \\
& 95 & 5.084 & 5.080 & 5.081 & 0.06 & -0.02 \\
& 97 & 4.113 & 4.119 & 4.116 & -0.07 & 0.09 \\
& 99 & 2.673 & 2.813 & 2.813 & -4.98 & 0.00 \\
& & & & & & \\
& 85 & 6.337 & 6.336 & 6.337 & 0.00 & 0.00 \\
& 87 & 6.323 & 6.321 & 6.321 & 0.03 & 0.01 \\
& 89 & 6.284 & 6.280 & 6.281 & 0.05 & 0.00 \\
91 & 6.194 & 6.187 & 6.187 & 0.11 & 0.00 \\
& 93 & 6.004 & 5.999 & 6.000 & 0.07 & -0.01 \\
& 95 & 5.646 & 5.670 & 5.671 & -0.44 & 0.00 \\
& 97 & 5.028 & 5.167 & 5.167 & -2.69 & 0.01 \\
99 & 4.050 & 4.489 & 4.489 & -9.78 & 0.01 \\
\hline
\end{tabular}

\section{Exhibit 15}

Comparison between the adjusted probability method and corrected-continuous method for reduced monitoring frequency. The option parameters are $S_{0}=100, K=100, \sigma=30 \%$, $r=10 \%$, and $T=0.2$. 

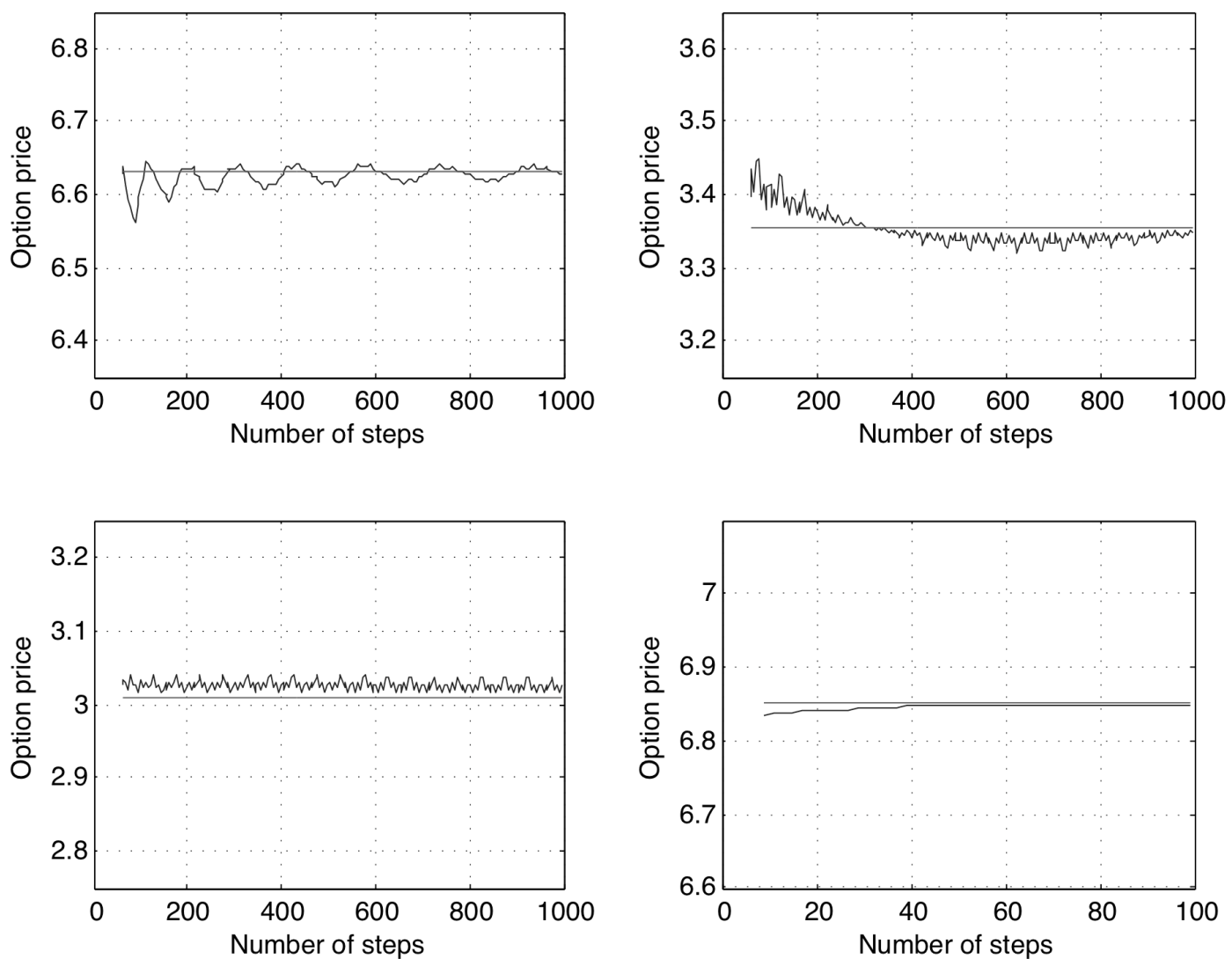

Exhibit 16

Panels (a)-(c) show convergence plots for various starting barrier levels with weekly monitoring. The parameters are taken from Table 2 in Duan et al. (2003): $S_{0}=100, K=100$, $\sigma=20 \%, r=10 \%, T=0.5$ and $\lambda=1.2$.

Panel (d) shows a comparison with the method of Ahn et al. (1999). The parameters for this calculation are taken to be $S_{0}=40, K=40, \sigma=40 \%, r=4.88 \%, T=1, L=35$ and a single monitoring time at $T=1 / 2$. 


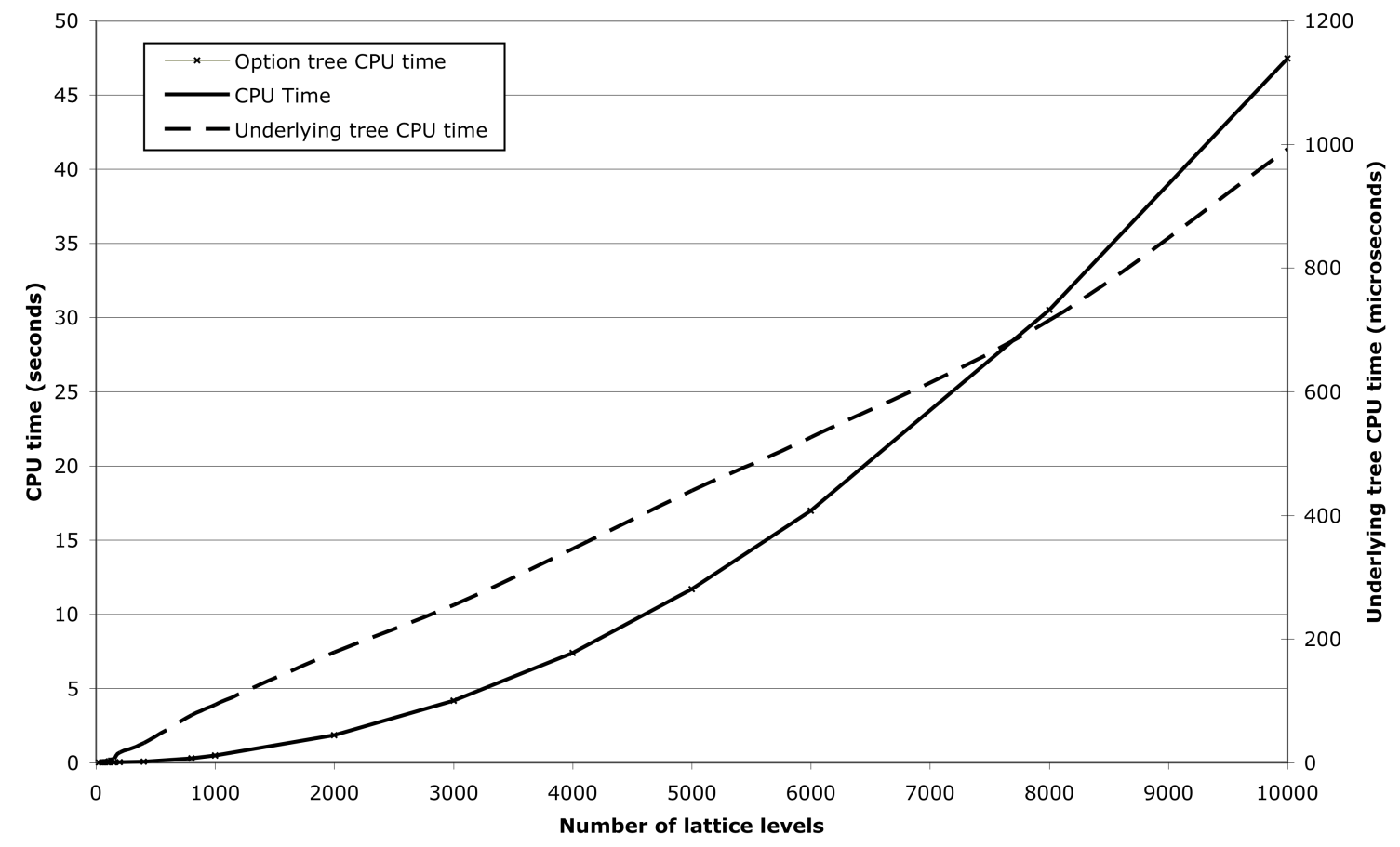

Exhibit 17

CPU times required for the underlying asset lattice and the option price tree 
Ahn, D. H. and Figlewski, S. and Gao, B. (1999). Pricing Discrete Barrier Options with an Adaptive Mesh Model, Working Paper.

Baldi, P. and Caramellino, L. and Iovino, M. G. (1999). Pricing general barrier options: a numerical approach using sharp large deviations. Mathematical Finance. 9, 293-322.

Boyle, P. and Lau, S. (1994). Bumping up against the barrier with the binomial method. Journal of Derivatives. 1, 6-14.

Broadie, M. and Glasserman, P. and Kou, S. G. (1997). A continuity correction for discrete barrier options. Mathematical Finance. 1(4): 325-348.

Broadie, M., Glasserman, P. and Kou, S.G. (1999). Connecting discrete and continuous pathdependent options. Finance and Stochastics. 3(1): 55-82.

Costabile, M. (2002). Extending the Cox-Ross-Rubinstein algorithm for pricing options with exponential boundaries. Proceedings of Algoritmy Conference on Scientific Computing, 2332.

Cox, J. C. and Ross, S. A. and Rubinstein, M. (1979). Option pricing: a simplified approach. Journal of Financial Economics. 7, 229-263.

Duan, J. C. and Dudley, E. and Gauthier, G. and Simonato, J. G. (2003). Pricing Discretely Monitored Barrier Options by a Markov Chain. Journal of Derivatives. 10(4), 9-31.

Figlewski, S. and Gao, B. (1999). The adaptive mesh model: a new approach to efficient option pricing. Journal of Financial Economics. 53, 313-351.

Geman, H. and Yor, M. (1996). Pricing and hedging double-barrier options: A probabilistic approach. Mathematical Finance. 6, 365-378. 
Kamrad, B. and Ritchken, P. (1991). Multinomial approximating models for options with k state variables. Management Science. 37, 1640-1652.

Kunitomo, N. and Ikeda, M. (1992). Pricing Options with Curved Boundaries. Mathematical Finance 2, 4, 275-298.

Pelsser, A. (2000). Pricing double barrier options using Laplace transforms. Finance and Stochastics. 4, 95-104.

Ritchken, P. (1995). On pricing barrier options. Journal of Derivatives. 3 (2), 19-28. 


\section{Endnotes}

The authors thank Stephen Figlewski, the editor, for valuable comments and the FinRisk for its financial support.

${ }^{1}$ Although we implement the transition probability adjusted model on a trinomial tree, the model can easily be implemented on the standard binomial lattice with slightly less effort. In Exhibit 5 and Exhibit 8 we show results for both models.

${ }^{2} \lambda \geq 1$. The special case of $\lambda=1$ makes the trinomial tree to collapse into a binomial tree.

${ }^{3}$ In the case of a down-and-in call option, the value of the call option is set to zero if the underlying price does not touch the barrier. 